\title{
Objetivo de Desarrollo Sostenible no 12: Consumo y Producción Sostenible. Estudio sobre hábitos de consumo de los estudiantes
}

\author{
Mónica Fernández-Morilla \\ Departamento de Educación. Universitat Internacional de Catalunya. c/ Terré 11-19, \\ 08017, Barcelona. España.mfernandezm@uic.es \\ ORCID: https://orcid.org/0000-0003-4176-1714
}

Ma Yolanda Fernández-Ramos

Departamento de Ciencias Sociales. Universidad Europea Miguel de Cervantes. c/Padre Julio Chevalier, 2, 47012, Valladolid. España. yolandafera@gmail.com

ORCID:https://orcid.org/0000-0002-1638-0752

Salvador Vidal-Ramèntol

Departamento de Educación. Universitat Internacional de Catalunya. c/ Terré 11-19, 08017, Barcelona.España.svidal@uic.es ORCID: http://orcid.org/0000-0002-2355-0668

\author{
Silvia Albareda-Tiana \\ Departamento de Educación. Universitat Internacional de Catalunya. c/ Terré 11-19, \\ 08017, Barcelona. España. salbareda@uic.es \\ ORCID: http://orcid.org/0000-0001-5643-8667
}

[Recibido: 15 Noviembre 2018. Revisado: 12 Enero 2019. Aceptado: 15 Enero 2019]

\begin{abstract}
Resumen: La consecución del Objetivo de Desarrollo Sostenible no 12 recogido en la Agenda 2030 de las Naciones Unidas supone la transición hacia pautas de consumo más sostenibles. El papel de la educación es clave para promover reflexión e inspirar hábitos de consumo que impliquen cambios relevantes en la manera en que usamos los recursos. En este sentido, resulta clave la formación inicial de los maestros, ya que éstos, serán un referente para la sociedad y los responsables de la formación escolar de los futuros profesionales. Así pues, el objetivo de este trabajo fue la realización de un diagnóstico sobre los hábitos de consumo de nuestros estudiantes, futuros maestros de Educación Primaria (GEP), a fin de poder desarrollar, en un futuro, un programa formativo que incida, especialmente, en los puntos más débiles detectados.
\end{abstract}

Palabras clave: Educación para la Sostenibilidad; Objetivos de Desarrollo Sostenible; hábitos de consumo; Grado en Educación Primaria

Sustainable Development Goal No. 12: Sustainable Consumption and Production. Study on student's consumption habits.

Abstract: The achievement of Sustainable Development Objective No. 12 included in the 2030 Agenda of the United Nations means the transition towards more sustainable patterns of consumption. The role of education is essential to promote reflection and inspire consumption habits that induce relevant changes on the way to using resources. In this sense, the initial training of teachers is a key issue; they will be a reference for society and those responsible for the school training of future professionals. Therefore, the objective of this work was to make a diagnosis about the consumption habits of our students, future teachers of Primary Education (GEP), in order to develop, in the future, a training program that influence, especially, the weakest points detected.

Keywords: Education for Sustainability; Sustainable Development Goals; Consumption habits; Primary Education Degree 
Para citar este artículo: Fernández-Morilla, M., Fernández-Ramos, M. Y., Vidal-Ramèntol, S. y AlbaredaTiana, S. (2019). Objetivo de Desarrollo Sostenible no 12: Consumo y Producción Sostenible. Estudio sobre hábitos de consumo de los estudiantes. Revista de Educación Ambiental y Sostenibilidad 1(1), 1201. doi: 10.25267/Rev_educ_ambient_sostenibilidad.2019.v1.i1.1201

\section{Introducción}

En noviembre del 2017, 15.364 científicos de 184 países han escrito una advertencia dirigida a toda la Humanidad, sobre la degradación ambiental del planeta, que afecta a la salud humana y a la propia supervivencia de la Tierra como planeta (Ripple et al., 2017). El manifiesto elaborado por la Comunidad Científica Mundial ha sido calificado como Segundo Aviso, puesto que ratifica y realiza un balance sobre los cambios transcurridos desde el primer informe La Advertencia de los Científicos del Mundo a la Humanidad escrito hace 25 años, por la asociación norteamericana Union of Concerned Scientists y más de 1500 científicos independientes, incluyendo la mayoría de los Premios Nobel en Ciencias que vivían entonces. En 1992 los científicos autores del manifiesto avisaron que sería necesario un gran cambio en nuestra forma de cuidar la Tierra y la vida sobre ella, si se quería evitar una enorme miseria humana (World Scientists' Warning to Humanity, 1992).

Informes científicos (IPCC, 2018; Landrigan et al. 2017; WWF, 2016) han contribuido a la comprensión de la fragilidad de los ecosistemas y a la interdependencia de las acciones humanas con la naturaleza y entre sí, así como las repercusiones de la salud ambiental en la salud humana. Ante las alarmantes evidencias sobre la situación de deterioro del planeta, en septiembre de 2015 Naciones Unidas aprueba la Agenda 2030 de los Objetivos de Desarrollo Sostenible (ODS), con la intención de concienciar a la ciudadanía global a un cambio de rumbo para lograr un futuro más sostenible y seguro.

Los ODS de la Agenda 2030 sustituyen a los Objetivos del Milenio y recogen los grandes retos que la humanidad ha de priorizar hasta el año 2030 para poder salvar la Tierra. Entre estos objetivos el no12 propone: "Garantizar modalidades de consumo y producción sostenibles" (Naciones Unidas, 2015), como meta para reflexionar y cambiar hacia modos de vida más austeros y menos consumistas.

El ODS no 12 supone revisar los patrones de producción y de consumo como estrategia que contribuya a la reducción de la pobreza y el hambre, y a la mejora de la calidad ambiental del planeta. El papel de la educación es clave para promover reflexión e inspirar hábitos de consumo que impliquen cambios relevantes en la manera en que usamos los recursos de la Tierra. En la reciente publicación de UNESCO sobre cómo impulsar los ODS a través de la educación, se afirma que esta no es sólo una parte esencial del desarrollo sostenible, sino también un instrumento clave para conseguirlo. Por esto, la educación supone una estrategia esencial en la consecución de los ODS (UNESCO, 2017).

Los graves problemas ecológicos que padece el planeta llevan a considerar que no se puede seguir consumiendo al mismo ritmo que hasta ahora. Ya nadie niega que la crisis ambiental se deba al modelo consumista de los países de Occidente. Si queremos frenar el deterioro del planeta, tendremos que cambiar los hábitos de vida de los habitantes que más consumen. El $15 \%$ de la población mundial que vive en los países de altos ingresos, es responsable del $56 \%$ del consumo total del mundo, sin 
embargo, el 40 \% más pobre, es responsable, tan sólo, del $11 \%$ (Naciones Unidas, 2002).

¿Pero cómo trasformar el ODS no 12 de producir y consumir de manera sostenible en una oportunidad para cambiar la mirada y ver la Tierra como la casa común? Para muchas personas el consumo sostenible, supone una restricción más que una oportunidad y no quieren cambiar el propio estilo de vida en el que se asocia la capacidad de consumir con la felicidad. Se requiere un cambio de paradigma para desarrollar unos hábitos de consumo que incrementen la calidad de vida y se ajusten a las necesidades reales de las personas y del planeta, provocando el mínimo de impacto ambiental y los máximos beneficios sociales posibles. Este cambio de paradigma solo puede darse en nuestras sociedades mediante la educación y el aprendizaje (UNESCO, 2014).

Si la educación es el instrumento imprescindible para cambiar la sociedad, hacia un modelo más sostenible, surge la cuestión: ¿Tienen las universidades la responsabilidad de participar activamente en esta educación para el desarrollo sostenible (EDS) y promocionar los ODS?

Los ODS incluyen una compleja gama de desafíos sociales, ecológicos y económicos, que se deben trabajar de manera integral y las universidades deben ser protagonistas para provocar el cambio hacia sociedades más justas y sostenibles, pero la cuestión es cómo integrar los ODS en el currículo universitario de manera que la educación sea transformativa y no meramente informativa.

Desarrollar un currículum donde la sostenibilidad sea un tema transversal, no equivale tanto a introducir contenidos medioambientales en la docencia (Barrón, Navarrete y Ferrer-Balas, 2010), sino más bien a formar personas que sepan analizar críticamente las interrelaciones entre los aspectos ambientales, sociales y económicos, de tal manera que en sus decisiones busquen las opciones más sostenibles y socialmente responsables, lo cual conduce a pensar en la repercusión ética de las propias acciones (Burgui y Chuvieco, 2017).

La transición hacia pautas de consumo más sostenibles está relacionada con la forma de entender el mundo, la cooperación, la equidad y en definitiva el propio estilo de vida. La actual crisis global (económica, ecológica y social) supone un desafío y una llamada a vivir de una manera diferente. Según los Informes Planeta Vivo (WWF, 2012, 2014, 2016) vivimos en un planeta, pero consumimos un planeta y medio. La globalización no ha supuesto una globalización de los recursos para todos. Las zonas ecológicamente más ricas de la Tierra son las que más se han deteriorado por el consumo excesivo de los países industrializados. La gran injusticia y desequilibrio social, consiste precisamente que sólo unos pocos consumen la mayoría de los recursos. La distribución de los bienes de la tierra en lugar de globalizarse, esto es, de compartir el planeta de una forma equitativa y justa, se ha descompensado aún más. Según el último informe de la Huella Ecológica Global, los países que más hectáreas consumen por ciudadano son Emiratos Árabes, Qatar, Bahréin, Dinamarca, Bélgica y Estados Unidos, los cuales necesitan más de 5 hectáreas por habitante. Mientras que en países subsaharianos como Zambia, Burundi y Eritrea o asiáticos como India y Sri Lanka sus habitantes sólo consumen 1 hectárea (Global Footprint Network, 2018). 
Este y otros muchos datos sobre la degradación del ambiente natural y sus consecuencias sociales, son un imperativo ético para la EDS. Esta concepción ética del consumo es especialmente importante en la formación de futuros profesionales, así como de líderes sociales de distinto tipo (Chuvieco, Burgui-Burgui, Da Silvia, Hussein, y Alkaabi, 2018).

En esta formación de futuros profesionales, resulta clave la formación inicial de los futuros maestros ya que éstos, son un referente para la sociedad. Para que ellos contribuyan a la construcción de una sociedad más sostenible en el ejercicio de su profesión docente, deben tener no sólo conocimientos y argumentos, sino también una conducta coherente con lo que enseñan en el aula. Así pues, y teniendo muy presente que la educación en el consumo responsable se considera clave en la educación ambiental (Wilk, 2002) y educación para la sostenibilidad (Fernández, Alfrez, Vidal, Fernández y Albareda, 2016; Fernández, Albareda y Vidal, 2018), el objetivo de este trabajo fue la realización de un diagnóstico sobre los hábitos de consumo de nuestros estudiantes, futuros maestros de Educación Primaria (GEP), a fin de poder desarrollar, en un futuro, un programa formativo que incida, especialmente, en los puntos más débiles detectados.

\section{Material y Método}

\section{Recogida y análisis de datos}

El test utilizado para la recogida de datos (diagnóstico) se puede consultar en la tabla 1. Este test es una adaptación sencilla realizada por los autores a partir de la encuesta de hábitos y valores sobre medio ambiente y sostenibilidad del Ayuntamiento de Barcelona y el Instituto de Gobierno y Políticas Públicas (Fernández, Albareda y Vidal, 2018). Cuenta con un total de 14 preguntas, de modo que las doce primeras proporcionan datos cuantitativos en una escala numérica de 0-3 (0: nunca, 1: alguna vez, 2: normalmente, 3: siempre) y las dos restantes, incluyen respuestas cualitativas de dos tipos, respuesta única entre diferentes opciones proporcionadas y respuesta abierta.

Tabla 1. Cuestionario utilizado para el diagnóstico de los hábitos de consumo de los estudiantes (realización propia a partir de la encuesta desarrollada por el Ayuntamiento de Barcelona y el Instituto de Gobierno y Políticas Públicas; Fernández, Albareda y Vidal, 2018).

\begin{tabular}{|c|}
\hline Coloca el $n^{\circ}$ correspondiente: Siempre 3 , Normalmente 2 , Alguna vez 1, Nunca 0 \\
\hline 1. ¿Reciclas residuos domésticos? \\
\hline 2. ¿Reciclas materia orgánica? \\
\hline 3. ¿Tratas de imprimir solamente lo esencial? \\
\hline 4. ¿Depositas la ropa usada en contenedores especiales? \\
\hline 5. ¿Depositas las pilas usadas y aparatos eléctricos en puntos especiales de recogida? \\
\hline 6. ¿Llevas los medicamentos usados al Centro de Salud o a puntos de recogida? \\
\hline 7. ¿Intentas reducir el consumo de agua en casa? \\
\hline 8. ¿Tratas de ahorrar calefacción en invierno? \\
\hline 9. ¿Apagas las luces cuando no son necesarias? \\
\hline 10. ¿Compras productos a granel? \\
\hline 11. ¿Evitas verter productos contaminantes al agua? \\
\hline 12. ¿Utilizas aparatos eléctricos de bajo consumo? \\
\hline
\end{tabular}




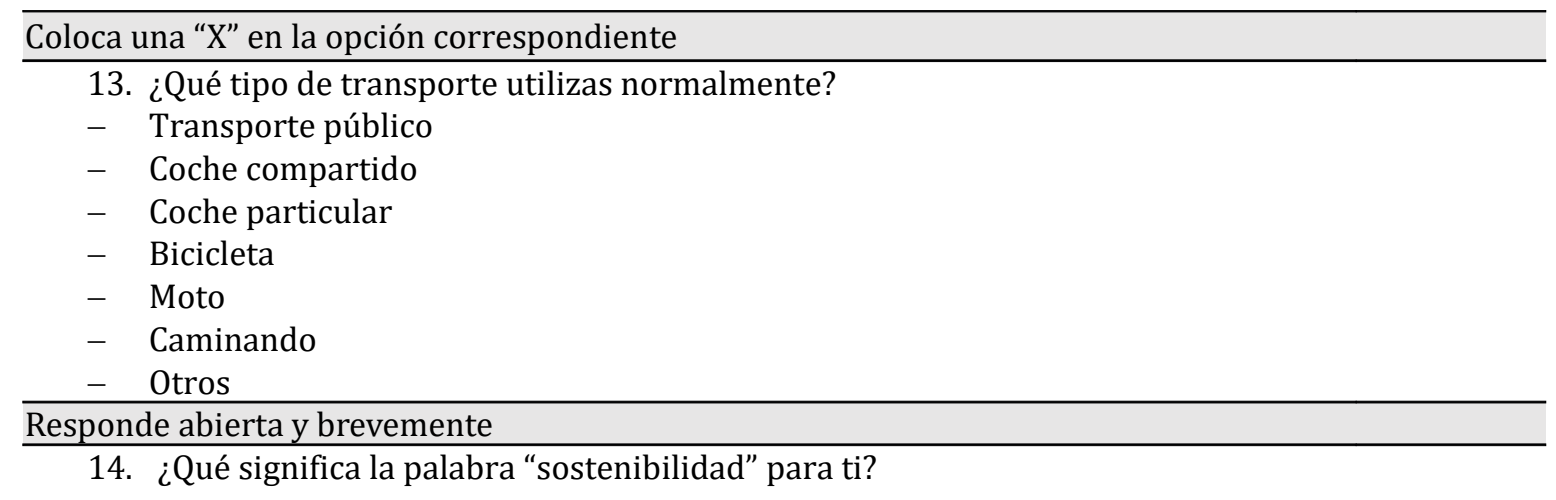

Se realizó un análisis cuantitativo de los datos obtenidos a través de los programas informáticos SPSS v.22.00 y Excel 2.3.

\section{Muestra}

El diagnóstico se llevó a cabo durante el primer semestre del curso 2017-2018. Los grupos de estudiantes del GEP de la Universitat Internacional de Catalunya fueron los estudiantes de 3o curso (Grupo A; $n=29$ estudiantes) y de $2^{\circ}$ curso (Grupo B; $n=27$ ). El hecho de analizar los hábitos de consumo en dos grupos separados, según el curso académico, permitirá planificar de forma independiente, la formación curricular específica para trabajar las conductas menos sostenibles -coincidentes o no- que muestren los estudiantes.

\section{Resultados}

\section{Diagnóstico de hábitos}

Tal y como se puede observar en la tabla 2, los estudiantes declararon, tanto en el grupo A como en el grupo B que los hábitos sostenibles que más realizan son:

1. Apagar las luces cuando no se necesitan.

2. Imprimir lo imprescindible.

3. Ahorrar calefacción en invierno.

4. Depositar residuos especiales como pilas y aparatos eléctricos en contenedores específicos para ellos.

En el caso del primer indicador, apagar la luz cuando no se necesitan, el valor de la mediana $\left(\mathrm{M}_{\mathrm{e}}\right)$ se situó en 3 para ambos grupos (A y B) y con una dispersión baja $\left(\mathrm{SD}_{\mathrm{A}}\right.$ $=0,47 ; \mathrm{SD}_{\mathrm{B}}=0,742$ ), lo que indica que los encuestados, en su mayoría, lo realizan siempre.

Respecto al segundo punto, imprimir lo imprescindible, la mediana varió entre 2,5 para el grupo $A\left(\mathrm{SD}_{\mathrm{A}}=0,841\right)$ y 3 para el grupo $\mathrm{B}\left(\mathrm{SD}_{\mathrm{B}}=0,802\right)$. Luego los datos sitúan el nivel de cumplimiento de este indicador entre prácticamente siempre y siempre respectivamente, para la mayoría de los encuestados.

En cuanto a ahorrar calefacción en invierno y depositar residuos especiales en contenedores específicos para ellos, la $\mathrm{M}_{\mathrm{e}}$ varió entre 3 para ambos indicadores en el 
grupo $\mathrm{A}\left(\mathrm{SD}_{\mathrm{A}}=0,928\right.$ y $\mathrm{SD}_{\mathrm{A}}=1,081$ respectivamente) y 2 para ambos indicadores en el grupo $\mathrm{B}\left(\mathrm{SD}_{\mathrm{B}}=0,997\right.$ y $\mathrm{SD}_{\mathrm{B}}=1,137$ respectivamente), es decir, que en el primer caso se trata de hábitos que se realizan siempre para la mayoría de los encuestados y en el segundo, se realizan normalmente.

Estos resultados parecen indicar que, en relación a estas cuatro conductas, no parece urgente una intervención educativa y de forma indirecta, los números muestran que los estudiantes son conocedores de las consecuencias de sus acciones y el impacto que estas tienen sobre el entorno.

Sin embargo, los hábitos sostenibles que declaran realizar en menor medida son:

1. Depositar los medicamentos caducados en contenedores especiales.

2. Llevar la ropa a contenedores para reciclar.

3. Comprar productos a granel.

En el caso del primer punto, depositar los medicamentos caducados en contenedores especiales, el resultado obtenido parece alarmante, ya que la mayoría de los encuestados han respondido nunca en ambos grupos y con una dispersión similar $\left(\mathrm{M}_{\mathrm{e}}\right.$ $=0$ para $\mathrm{A}$ y $\mathrm{B} ; \mathrm{SD}_{\mathrm{A}}=1,280 ; \mathrm{SD}_{\mathrm{B}}=1,188$ ).

En los dos puntos siguientes, llevar ropa a contenedores especiales para su reciclaje y comprar productos a granel, la respuesta mayoritaria ha sido nunca en el caso del grupo $\mathrm{A}\left(\mathrm{M}_{\mathrm{e}}=0\right.$ para ambos indicadores; $\mathrm{SD}_{\mathrm{A}}=1,195$ y SD $=0,686$ respectivamente $)$ o muy ocasionalmente en el caso del grupo $\mathrm{B}\left(\mathrm{M}_{\mathrm{e}}=1\right.$ para ambos indicadores; $\mathrm{SD}_{\mathrm{B}}=$ 1,074 y $\mathrm{SD}_{\mathrm{B}}=0,859$ respectivamente).

Estos resultados indican la necesidad urgente de incidir mediante un programa educativo en las conductas relacionadas especialmente con estos indicadores.

En una posición intermedia entre ellos, con un nivel de cumplimiento, por parte de la mayoría de los encuestados en el caso de los dos grupos (A y B) de "normalmente" $\left(\mathrm{M}_{\mathrm{e}}=2\right.$ para todos los indicadores $)$ se situaron los siguientes hábitos:

1. Reciclar materia orgánica $\left(\mathrm{SD}_{\mathrm{A}}=1,298 ; \mathrm{SD}_{\mathrm{B}}=1,182\right)$

2. Reciclar residuos domésticos $\left(\mathrm{SD}_{\mathrm{A}}=1,081 ; \mathrm{SD}_{\mathrm{B}}=0,93\right)$

3. Evitar verter residuos contaminantes por la red de aguas $\left(\mathrm{SD}_{\mathrm{A}}=1,125 ; \mathrm{SD}_{\mathrm{B}}=\right.$ 1,141)

4. Utilizar electrodomésticos de bajo consumo $\left(\mathrm{SD}_{\mathrm{A}}=1,018 ; \mathrm{SD}_{\mathrm{B}}=0,759\right)$

5. Intentar reducir el consumo de agua del hogar $\left(\mathrm{SD}_{\mathrm{A}}=1,091 ; \mathrm{SD}_{\mathrm{B}}=1,155\right)$

Estos resultados parecen indicar un nivel aceptable de compromiso y, por tanto, hábitos bastante integrados en las conductas de los grupos en estudio. 
Tabla 2. Estadísticos descriptivos comparativos de los dos grupos (A y B). Me: Mediana; SD: Desviación Estándar

\begin{tabular}{lcccrr}
\hline & Grupo & N & $\mathbf{M}_{\mathbf{e}}$ & Media & SD \\
\hline 1. ¿Reciclas residuos domésticos? & $\mathrm{A}$ & 29 & 2 & 1,90 & 1,081 \\
& $\mathrm{~B}$ & 27 & 2 & 1,89 &, 934 \\
\hline 2. ¿Reciclas materia orgánica? & $\mathrm{A}$ & 29 & 2 & 1,55 & 1,298 \\
& $\mathrm{~B}$ & 27 & 2 & 1,63 & 1,182 \\
\hline 3. ¿Tratas de imprimir solamente lo esencial? & $\mathrm{A}$ & 29 & 2,5 & 2,28 &, 841 \\
& $\mathrm{~B}$ & 27 & 3 & 2,48 &, 802 \\
\hline 4. ¿Depositas la ropa usada en contenedores especiales? & $\mathrm{A}$ & 29 & 0 & 1,00 & 1,195 \\
& $\mathrm{~B}$ & 27 & 1 & 1,00 & 1,074 \\
\hline 5. ¿Depositas las pilas usadas y aparatos eléctricos en puntos especiales & $\mathrm{A}$ & 29 & 3 & 2,10 & 1,081 \\
de recogida? & $\mathrm{B}$ & 27 & 2 & 1,70 & 1,137 \\
\hline 6. ¿Llevas los medicamentos usados al Centro de Salud o a puntos de & $\mathrm{A}$ & 29 & 0 &, 93 & 1,280 \\
recogida? & $\mathrm{B}$ & 27 & 0 &, 89 & 1,188 \\
\hline 7. ¿Intentas reducir el consumo de agua en casa? & $\mathrm{A}$ & 29 & 1 & 1,24 & 1,091 \\
& $\mathrm{~B}$ & 27 & 2 & 1,89 & 1,155 \\
\hline 8. ¿Tratas de ahorrar calefacción en invierno? & $\mathrm{A}$ & 29 & 3 & 2,17 &, 928 \\
& $\mathrm{~B}$ & 27 & 2 & 1,93 &, 997 \\
\hline 9. ¿Apagas las luces cuando no son necesarias? & $\mathrm{A}$ & 29 & 3 & 2,69 &, 471 \\
& $\mathrm{~B}$ & 27 & 3 & 2,63 &, 742 \\
\hline 10. ¿Compras productos a granel? & $\mathrm{A}$ & 29 & 0 &, 55 &, 686 \\
& $\mathrm{~B}$ & 27 & 1 &, 74 &, 859 \\
\hline 11. ¿Evitas verter productos contaminantes al agua? & $\mathrm{A}$ & 29 & 2 & 1,86 & 1,125 \\
& $\mathrm{~B}$ & 27 & 2 & 1,93 & 1,141 \\
\hline 12. ¿Utilizas aparatos eléctricos de bajo consumo? & $\mathrm{A}$ & 29 & 2 & 1,59 & 1,018 \\
& $\mathrm{~B}$ & 27 & 2 & 2,04 &, 759 \\
\hline
\end{tabular}

En relación a la pregunta 13: ¿Qué tipo de transporte utilizas normalmente? En la figura 1 ( $\mathrm{a}$ y b) se recogen las respuestas de los estudiantes. Tal y como se puede observar, en el primer caso, grupo A, el trasporte público y el coche de uso individual se situaron a un mismo nivel con un $26 \%$ de las respuestas, seguidos de un $24 \%$ de estudiantes que comparten coche, $15 \%$ caminando, $7 \%$ que usan moto y un $2 \%$ en bicicleta. En el segundo, grupo B, el trasporte público representó el $43 \%$ de las respuestas, seguido de un $25 \%$ de estudiantes que se desplazan a pie, un $13 \%$ en moto, $12 \%$ en coche compartido y un $7 \%$ en coche individual. Ningún estudiante manifestó desplazarse en bicicleta.

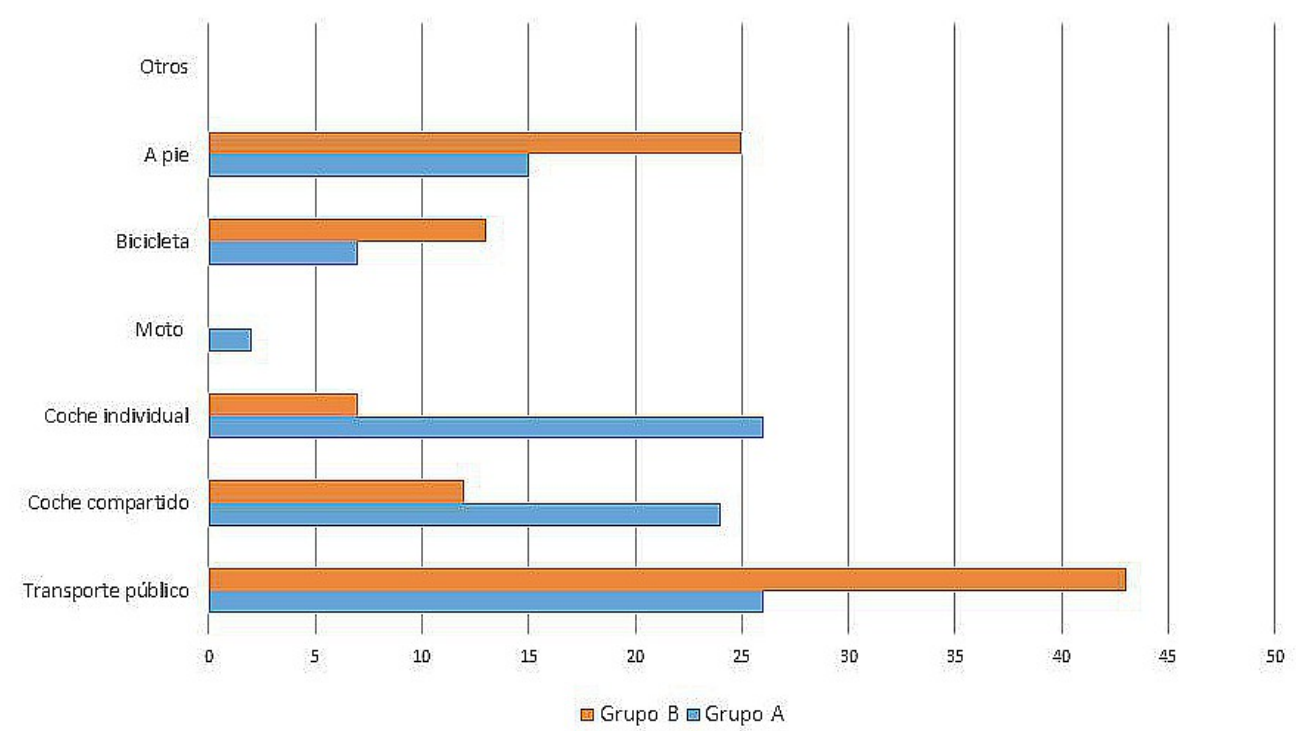

Figura 1. Respuestas de los estudiantes ante la pregunta ¿Qué tipo de transporte utilizas normalmente? 
Finalmente, en la tabla 3 se recogen las respuestas recibidas a la pregunta ¿Qué significa para ti la palabra "sostenibilidad"?

Tabla 3. Respuestas de los estudiantes a la pregunta no 14: ¿Qué significa para ti la palabra sostenibilidad?

Respuestas de grupo A

in

- El cuidado del medio ambiente para evitar su contaminación y destrucción.

- El hecho de respetar el medio ambiente.

- Usar los recursos para mantener el Planeta lo mejor posible.

- Es un acto de gratitud hacia la naturaleza, devolviéndole un trato justo por todo lo que nos da.

- Ser responsable con la Tierra pensando en el futuro.

- Mantener los recursos en equilibrio.

\section{Respuestas del grupo B}

\section{Discusión}

Nuestra sociedad, orientada al consumo, supone una enorme presión sobre la Tierra. Los ciudadanos de la Unión Europea constituyen menos del $10 \%$ de la población mundial y, sin embargo, consumen el $50 \%$ de la carne, el $25 \%$ del papel y el $15 \%$ de la energía (European Comission, 2010). En España la huella ecológica de su población indica que para mantener este ritmo de consumo necesitaríamos en torno a 4 hectáreas globales (hg) per cápita, mientras que, según la biocapacidad de la Tierra, tan sólo nos corresponderían 1,7 hg (WWF, 2016; Ministerio de Medio Ambiente y Medio Rural y Marino, 2008). En Cataluña, la diferencia entre la biocapacidad de su territorio y la huella ecológica de sus habitantes, muestra que existe un déficit ecológico promedio de más de $4 \mathrm{hg}$, con un déficit severo en la ciudad de Barcelona, la cual necesitaría una superficie 10 veces superior a ella para poder mantener su nivel de consumo (FACUA Andalucía-Consumidores en acción, 2009).

En consonancia con estos datos sobre consumo personal de los europeos y españoles en general, y catalanes en particular, los resultados obtenidos en este trabajo mostraron tres aspectos especialmente débiles en los hábitos de consumo de los estudiantes: depositar los medicamentos caducados en contenedores especiales, llevar la ropa a contenedores para su reciclaje y comprar productos a granel. Acciones que, en su mayoría, nunca realizaban.

Los medicamentos caducados representan un factor altamente contaminante para los sitios de disposición final de Residuos Sólidos Urbanos (RSU). Existen estudios que demuestran que el 58,3 \% de los medicamentos caducados y eliminados como RSU, se podrían aprovechar para terapéutica veterinaria, $\mathrm{y}$, además, desde una perspectiva ambiental, la disminución de la contaminación por el vertido de medicamentos, favorecía la biodegradación de los vertederos a cielo abierto y aumentaría la rapidez de generación de biogás (Kiss y Encarnación, 2006).

Además, España es un gran consumidor de medicamentos. En 2011, en el sistema público español de salud se habían extendido más de 1.000 millones de recetas, una 
media de 21 recetas por persona/año (Federación Empresarial de Farmacéuticos Españoles, 2017). Dada esta circunstancia, y para promover el reciclaje de los medicamentos caducados en España, el sector farmacéutico creó un Sistema Integrado de Gestión y Recogida de Envases -SIGRE-, cuyo objetivo es dar ese tratamiento medioambiental específico a los residuos de medicamentos de origen domiciliario y a sus envases (SIGRE, 2016). Es por ello que muchos centros de salud y farmacias disponen de los denominados puntos SIGRE que proporcionan contenedores específicos donde se pueden depositar estos medicamentos. Es posible que los estudiantes no tuvieran información de esta iniciativa que lleva más de una década en marcha.

Por otra parte, y en relación con la compra de productos a granel, comprar la cantidad que se necesita de un alimento, permite ahorrar dinero, pero también, evita el desperdicio de los mismos. Se estima que cada año se desaprovechan más de 1.300 millones de toneladas de alimentos en buen estado en el mundo, 89 millones de toneladas en Europa y 8 millones en España (European Comission-BIO Intelligence Service, 2010). Así mismo, disminuiría el consumo de petróleo y se eliminarían las emisiones de $\mathrm{CO}_{2}$ y otros gases de efecto invernadero generados en la fabricación de los envases. De acuerdo con la Environmental Protection Agency, que promociona los programas Green Lights y Energy Star, cada kilovatio - hora de electricidad que no se

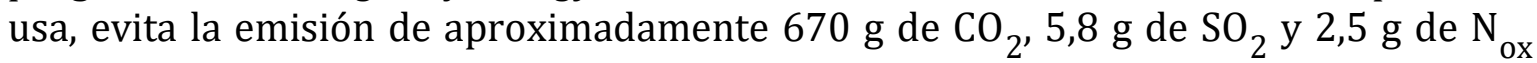
(Como se cita en: Gobierno de Chile, 2002). Además, se evitarían las emisiones derivadas del transporte a larga distancia de los productos manufacturados con la reducción del uso de energía en la distribución (Palmarola, 2015) y se potenciaría el desarrollo del comercio local.

En relación al reciclado de la ropa usada, es importante señalar que el sector de la moda ocupa el tercer lugar en las prioridades de los jóvenes europeos después de la alimentación y el ocio con amigos (Generalitat de Catalunya, 2013). De todos los países analizados, España y El Reino Unido son los que tienen el mayor número de jóvenes que gastan entre 50 y 80 euros al mes en moda. Este consumo se ha visto favorecido por el gran crecimiento de la industria textil y la tendencia fast fashion de las grandes marcas del mundo de la moda, las cuales cambian su oferta y lanzan nuevas colecciones en lapsos de tiempo muy cortos y con precios más asequibles (López, 2012). Este elevado nivel de consumo lleva asociado la generación de grandes cantidades de residuos, tanto pre como post consumo (Pensupa et al., 2017). La industria textil es una de las más contaminantes por los productos químicos y las grandes cantidades de agua que requiere, así mismo entre el 10-20\% de todos los productos textiles son restos de fabricación que se desperdician (TextileExchange, 2012). A nivel post consumo los restos de ropas y tejidos son una mezcla de fibras naturales, fibras sintéticas y otras sustancias como botones, cremalleras...que hace muy difícil su degradación (Payne, 2015). Estos restos pueden ser reciclados, pero de los 13 millones de toneladas contabilizadas en 2010 sólo un 15\% fue recuperado (Joung y Park-Poaps, 2013; Wallander, 2012).

En este trabajo, los estudiantes del grupo A manifestaron en su mayoría que nunca realizaban este gesto y los del grupo B, muy ocasionalmente. Es muy importante informar y concienciar a los jóvenes de la posibilidad de depositar la ropa usada en contenedores especiales para su posterior categorización y reciclaje a través de 
distintas vías, desde la reutilización de las prendas hasta la venta a las industrias de reciclaje de componentes o para fabricar ropa nueva (Bediako, Wei y Yun, 2016; Binici, Gemci, Aksogan y Kaplan, 2010).

El transporte por carretera en su modalidad de pasajeros, tanto del tipo privado como público, supone un sector de notable relevancia socioeconómica. Hay estimaciones que fijan su aportación al Producto Interior Bruto (PIB) de España en un 7\% como media (Sotelo, Sotelo y Toln, 2011), pero este hecho supone al mismo tiempo, un enorme coste en lo que a contaminación atmosférica se refiere (Gurjar, Butler, Lawrence y Lelieveld, 2008; Colvile et al., 2002). En cuanto a consumo energético, el consumo de los vehículos privados representa en España alrededor del 50\% del total del consumo de transporte por carretera, y sólo un 3\% se debe al transporte colectivo de pasajeros (Sotelo, Sotelo y Toln, 2011).

Aunque estos son los datos promedio para el país, los datos obtenidos en este trabajo muestran menor preferencia por el uso del vehículo privado respecto al transporte público, pero con grandes diferencias entre los dos grupos de estudiantes analizados. En el grupo A, la preferencia mayoritaria fue compartida entre el uso individual del coche y el transporte público, con un cuarto de representación cada uno. Sin embargo, en el grupo B, la mayoría de los estudiantes eligen habitualmente el transporte público, con un porcentaje muy bajo de aquellos que usaban el coche de forma individual.

En el caso del coche compartido, en España, la Dirección general de Tráfico (Ministerio del Interior) ha desarrollado un plan de fomento de esta alternativa en las grandes ciudades, Madrid y Barcelona, ofreciendo carriles especiales en las autopistas para una mayor descongestión del tráfico (carriles para Vehículos de Alta Ocupación -VAO-). Así mismo, se aplican descuentos de hasta el $40 \%$ en los peajes de algunas autopistas en el caso de que los vehículos contengan tres o más ocupantes, lo cual ha podido influir también en sus preferencias (Abertis Company, 2015).

Finalmente, y en relación a la pregunta de qué entienden por la palabra sostenibilidad las respuestas de los estudiantes de ambos grupos son similares; todas ellas incluyen los verbos cuidar, mantener, respetar en relación con el medio ambiente, nuestro Planeta o el mundo en el que vivimos. Parece que el estudiante no entiende que la palabra sostenibilidad trasciende a la de medio ambiente, ya que incluye la búsqueda de la calidad ambiental, pero también la equidad y la justicia social; implica un abanico de conocimientos y habilidades para la acción, que incluyan criterios éticos (Martínez-Agut, Aznar, Ull y Piero, 2007).

\section{Conclusión}

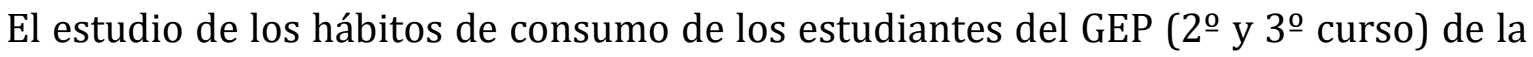
Universitat Internacional de Catalunya reveló que, si bien existen conductas sostenibles bastante integradas en su manera de vivir -ya que las desarrollan SIEMPRE O CASI SIEMPRE- como: apagar las lunes cuando no son necesarias, imprimir lo indispensable, ahorrar calefacción en invierno y depositar pilas y aparatos electrónicos en contenedores especiales, carecen de ciertos hábitos -y además coincidentes entre los dos grupos de estudiantes- que resultan fundamentales a la hora de disminuir el impacto sobre el Planeta tal y como se ha argumentado previamente. En este sentido, es alarmante el hecho de que los alumnos 
manifiesten que NUNCA o CASI NUNCA reciclan la ropa usada, compran productos a granel o depositan los medicamentos en contenedores especiales, con lo que parece urgente trabajar en estos puntos desde la educación.

Así mismo, sería interesante incidir en el uso de alternativas de transporte más sostenibles tales como la bicicleta; a penas, aparece representada. Pero, no parece que este sea un aspecto prioritario, ya que, el uso del transporte público junto con los desplazamientos a pie y el coche compartido, superaron notablemente al transporte en coche particular.

Finalmente, parece crucial trabajar en la comprensión del concepto sostenibilidad, ya que una visión integral de las tres dimensiones del mismo (no solo la medio ambiental), podría ayudar en la toma de decisiones en cuanto al consumo.

Tal y como se ha podido mostrar a través de este trabajo, un diagnóstico de estas características, permite sentar las bases sobre las que diseñar programas formativos que incidan, específicamente, en los puntos más débiles que se detecten en los grupos de estudio.

\section{Referencias}

Abertis Company (2015). Vehículos de Alta Ocupación (VAO). Recuperado de: https://www.autopistas.com/es/vao

Barrón, A., Navarrete, A. y Ferrer-Balas. D. (2010). Sostenibilización curricular en las universidades españolas ¿Ha llegado la hora de actuar? Revista Eureka sobre Enseñanza y Divulgación de las Ciencias, 7, 388-399.

Bediako J. K., Wei W, Yun Y. S. (2016) Low-cost renewable adsorbent developed from waste textile fabric and its application to heavy metal adsorption. Journal of the Taiwan Institute of Chemical Engineers, 63, 250-258.

Binici, H., Gemci, R., Aksogan, O. y Kaplan, H. (2010). Insulation properties of bricks made with cotton and textile ash wastes. International Journal of Materials Research, 101(7), 894-899.

Burgui, M. y Chuvieco, E. (2017). Dimensiones éticas en los dilemas ambientales: estudio de casos. Madrid: Ediciones Internacionales Universitarias.

Chuvieco, E., Burgui-Burgui, M., Da Silva, E. V., Hussein, K., y Alkaabi, K. (2018). Factors affecting environmental sustainability habits of university students: Intercomparison analysis in three countries (Spain, Brazil and UAE). Journal of Cleaner Production, 198, 1372-1380.

Colvile, R. N., Woodfield, N. K., Carruthers, D. J., Fisherd, B. E. A., Rickarde, A., Neville, S. y Hughes, A. (2002). Uncertainty in dispersion modelling and urban air quality mapping. Environmental Science \& Policy 5(3), 207-220.

European Commission (2010). Smarter and Cleaner. Recuperado de: http://ec.europa.eu/environment/eussd/pdf/brochure_scp.pdf

European Comission-BIO Intelligence Service (2010). Preparatory Study on Food Waste across EU 27 (Final Report). Recuperado de: http://ec.europa.eu/environment/eussd/pdf/bio_foodwaste_report.pdf 
FACUA Andalucía-Consumidores en acción (2009). Guía sobre la Huella Ecológica y hábitos de consumo responsable. Recuperado de: http://www.facua.org/es/guia.php?Id=105\&capitulo=886

Federación Empresarial de Farmacéuticos Españoles, (2017). El consumo de medicamentos en unidades sólo ha crecido un 2,5\% en 48 años. Observatorio del medicamento, sala de prensa.

Fernández, M., Alférez, A., Vidal, S., Fernández, M. Y., y Albareda, S. (2016). Methodological approaches to change consumption habits of future teachers in Barcelona, Spain: reducing their personal Ecological Footprint. Journal of Cleaner Production, 122, 154-163.

Fernández, M. Albareda, S. y Vidal, S. (2018). En el camino hacia el compromiso ambiental: estudio sobre hábitos de consumo personales. Temps d'Educació, 54, 146-160.

Generalitat de Catalunya (2013). El joves i el consum de roba i complements. Estudis sobre els hàbits de consum dels joves de Catalunya. Barcelona: Agència Catalana del Consum. Recuperado de: http://aplicacio.consum.gencat.cat/documentacio/15022.pdf

Gobierno de Chile (2002). Estudio de ciclo de vida de 12 envases y embalajes. Proyecto Minimización de Residuos provenientes de Envases y Embalajes. Recuperado de: http://infohouse.p2ric.org/ref/18/17615.pdf

Global Footprint Network (2018). On-line home of the Ecological Footprint. Recuperado de: http://data.footprintnetwork.org

Gurjar, B. R., Butler, T. M., Lawrence, M. G. y Lelieveld, J. (2008). Evaluation of emissions and air quality in megacities. Atmospheric Environment, 42(7), 15931606.

Intergovernmental Panel on Climate Change (IPCC) (2018). Global warming of $1.5{ }^{o} C$. Recuperado de: http://report.ipcc.ch/sr15/pdf/sr15_spm_final.pdf

Joung, H. M. y Park-Poaps, H. (2013). Factors motivating and influencing clothing disposal behaviours. International Journal of Consumer Studies, 37(1), 105-111.

Kiss, G. y Encarnación, G. (2006). Los productos y los impactos de la descomposición de residuos sólidos urbanos en los sitios de disposición final. Gaceta Ecológica, 79, 39-51. Recuperado de: http://www.redalyc.org/pdf/539/53907903.pdf

Landrigan, P. J., Fuller, R., Acosta, N. J., Adeyi, O., Arnold, R., Baldé, A. B., ... y Chiles, T. (2017). The Lancet Commission on pollution and health. The Lancet.

López, M. C. (2012). El futuro que queremos y las incidencias de la Fast Fashion. Arte \& Diseño, 10(1), 29-33.

Martínez-Agut, M. P., Aznar, P., Ull, M. A. y Piñero, A. (2007). Promoción de la sostenibilidad en los currícula de la enseñanza superior desde el punto de vista del profesorado: un modelo de formación por competencias. Educatio Siglo XXI, 25, 187-208

Ministerio de Medio Ambiente y Medio Rural y Marino (2008). Análisis de la Huella Ecológica de España. Sostenibilidad y Territorio. Gobierno de España. 
Recuperado

de:

https://www.asturias.es/medioambiente/articulos/ficheros/Huella

$\% 20$ ecologica\%20de\%20Espana.pdf

Naciones Unidas (2015). Transformando nuestro mundo: la Agenda 2030 para el Desarrollo Sostenible. Resolución aprobada por la Asamblea General de Naciones Unidas el 25 septiembre de 2015. Referencia A/70/L.1. Recuperado de: http://www.un.org/ga/search/view_doc.asp?symbol=A/70/L.1\&Lang=S

Naciones Unidas (2002). Cumbre de Johannesburgo. Nota informativa. Recuperado de: http://www.un.org/spanish/conferences/wssd/modelos_ni.htm

Palmarola, A. (2015). Eficiencia energética en flotas. VIII Congreso de AEGFA 2015 de Gestores de Flotas. Salón Internacional del Automóvil de Barcelona. Fira Barcelona-Montjüic (12 de mayo).

Payne, A. (2015). Open- and closed-loop recycling of textile and apparel products. En: S. S. Muthu (ed.). Handbook of life cycle assessment (LCA) of textiles and clothing. (pp 103-123). Cambridge, UK: Woodhead Publishing.

Pensupa, N., Leu, S. Y., Hu, Y., Du C., Liu, H., Jing, H., Wang, H. \& Lin, C. (2017) Recent Trends in Sustainable Textile Waste Recycling Methods: Current Situation and Future Prospects. Topics in Current Chemestry, 375(5). doi: 10.1007/s41061017-0165-0

Ripple, W. J., Wolf, C., Newsome, T. M., Galetti, M., Alamgir, M., Crist, E., ... y 15,364 scientist signatories from 184 countries. (2017). World scientists' warning to humanity: A second notice. BioScience, 67(12), 1026-1028.

SIGRE, Sistema Integrado de Gestión y Recogida de Envases (2016). Medicamento y Medio ambiente. Blog corporativo SIGRE [En-line]. Recuperado de: http://blogsigre.es/

Sotelo, J. A., Sotelo, M. y Tolón, A. (2011). Las emisiones de gases de efecto invernadero en el sector transporte por carretera. Investigaciones geográficas, $54,133-169$

TextileExchange (2012). 2012 Final Report. TextileExchange.org. Recuperado de: https://textileexchange.org/wp-content/uploads/2017/08/2012-Textile-

Exchange-Annual-Report.pdf

UNESCO (2014). Hoja de ruta para la ejecución del programa de acción mundial de Educación para el Desarrollo Sostenible. Paris. Recuperado de: http://unesdoc.unesco.org/images/0023/002305/230514s.pdf

UNESCO (2017). Education for Sustainable Development Goals. Learning Objectives. Paris. Recuperado

de: http://unesdoc.unesco.org/images/0024/002474/247444e.pdf

Wallander, M. (2 January, 2012). Why textile waste should be banned from landfills. TriplePundit [Media Platform]. Recuperado de: https://www.triplepundit.com/2012/01/textile-waste-be-banned-landfills/

Wilk, R. (2002). Consumption, human needs, and global environmental change. Global Environmental Change, 12, 5-13. 
World Scientists' Warning to Humanity (1992). Recuperado de: https://www.ucsusa.org/sites/default/files/attach/2017/11/World \%20Scientists\%27\%20Warning\%20to\%20Humanity\%201992.pdf

WWF (2012). Informe Planeta Vivo 2012. Recuperado de: http://www.wwf.es/noticias/informes_y_publicaciones/informe_planeta_vivo _2012/

WWF (2014). Informe Planeta Vivo 2014. Recuperado de: http://www.wwf.es/noticias/informes_y_publicaciones/informe_planeta_vivo /

WWF (2016). Informe Planeta Vivo 2016. Recuperado de: http://awsassets.wwf.es/downloads/informeplanetavivo_2016.pdf 